\title{
Influencing surgical management in patients with carcinoma of the cervix using a T2- and ZOOM-diffusion-weighted endovaginal MRI technique
}

\author{
K Downey ${ }^{1}, \mathrm{M} \mathrm{Jafar}{ }^{1}$, A D Attygalle ${ }^{2}$, S Hazell ${ }^{2}, \mathrm{~V}$ A Morgan ${ }^{1}, \mathrm{~S} \mathrm{~L} \mathrm{Giles}{ }^{1}, \mathrm{M} \mathrm{A} \mathrm{Schmidt}{ }^{1}, \mathrm{~T}$ E J Ind ${ }^{3}$, \\ $\mathrm{J} \mathrm{H}$ Shepherd $^{3}$ and N M deSouza*,1 \\ ${ }^{1}$ CRUK and EPSRC Cancer Imaging Centre, Institute of Cancer Research and Royal Marsden NHS Foundation Trust, Surrey SM2 \\ 5PT, UK; ${ }^{2}$ Department of Histopathology, Royal Marsden NHS Foundation Trust, Downs Road, Surrey SM2 5PT, UK and \\ ${ }^{3}$ Department of Gynecology, Royal Marsden NHS Foundation Trust, Downs Road, Surrey SM2 5PT, UK
}

Background: Endovaginal MRI (evMRI) at 3.0-T with T2-weighted (T2-W) and ZOnal Oblique Multislice (ZOOM)-diffusion-weighted imaging (DWI) potentially improves the detection of stage la/lb1 cervical cancer. We aimed to determine its sensitivity/specificity, document tumour-to-stromal contrast and establish the effect of imaging on surgical management.

Methods: Following ethical approval and written informed consent, 57 consecutive patients with suspected stage la/lb1 cervical cancer underwent evMRI at 3.0-T using T2-W and ZOOM-DWI. Sensitivity/specificity were calculated against histopathology for two independent observers. Tumour-to-stromal contrast was determined on T2-W, and diffusion-weighted $\left(b=800 \mathrm{smm}^{-2}\right.$ ) images and apparent diffusion coefficients (ADCs) were recorded. In patients due for radical vaginal trachelectomy (RVT), change of surgical management based on imaging findings was documented.

Results: Sensitivity/specificity for detecting tumour was the following: reporting read 88.0/81.8\%, anonymised read $92.0 / 81.8 \%$ (observer 1); 84.0/72.7\% (observer2; median tumour volume $=1.7 \mathrm{~cm}^{3}$ ). Intraobserver agreement was excellent $(\mathrm{kappa}=0.89$ ) and the interobserver agreement was good (kappa $=0.65$ ). Tumour-to-stromal contrast was greater on ZOOM-DWI compared with T2-W images $(3.35 \pm 2.36$ vs $1.39 \pm 0.95 ; P<0.0004)$. Tumour and stromal ADCs were significantly different $(P<0.00001)$. In 31 patients due for RVT, evMRI altered surgical management in 12 (38.7\%) cases (10 cone-biopsy, 2 chemoradiotherapy).

Conclusion: T2-W + ZOOM-DWI evMRI has high sensitivity/specificity for detecting stage la/lb1 cervical tumours; in patients due for RVT, the surgical management was altered in $\sim 39 \%$.

With the implementation of screening programmes, carcinoma of the cervix often presents with early-stage small volume disease, which may potentially be treated using fertility-sparing procedures. Trachelectomy is an option in fertile women $<45$ years of age with confirmed invasive cervical cancer stage Ia2/Ib (Abu-Rustum et al, 2008) where at least a 1-cm length of normal cervical canal exists between the superior tumour margin and the internal os (Shepherd et al, 2006; Gien and Covens, 2010). Accurate staging and depiction of tumour margins in relation to the normal cervical anatomy (Bipat et al, 2011), therefore, is crucial in selecting patients for trachelectomy. An MRI using T2-weighted (T2-W) sequences is the imaging technique of choice for visualising the cervix because of its soft-tissue contrast, and is routinely performed using external phased-array receiver coils on $1.5-\mathrm{T}$ and $3.0-\mathrm{T}$

*Correspondence: Professor NM deSouza; E-mail: nandita.deSouza@icr.ac.uk

Received 30 April 2013; revised 11 June 2013; accepted 22 June 2013; published online 18 July 2013

(c) 2013 Cancer Research UK. All rights reserved 0007-0920/13 
clinical systems. As it provides images with a $>1-\mathrm{mm}$ in-plane resolution and a 3- to 4-mm slice thickness, tumour dimensions from previous studies where it has achieved sensitivity/specificity for detecting tumour of $\sim 80 \%$ (Hricak et al, 2007) have been $>1 \mathrm{~cm}$ (Sahdev et al, 2007).

The spatial resolution of MR images may be improved by the use of receiver coils close to the region of interest because they provide significant increases in signal-to-noise ratio (SNR; deSouza et al, 1996; deSouza et al, 2000; deSouza et al, 2006). The use of an endovaginal receiver coil has previously been shown to improve the detection of tumours of $<1.0 \mathrm{~cm}^{3}$ on T2-W imaging (Soutter et al, 2004) and also allows detailed visualisation of the parametrium up to $6 \mathrm{~cm}$ from the surface of the internal coil (Gilderdale et al, 1999). We have previously demonstrated at 1.5-T that sensitivity improves further when T2-W imaging is supplemented with diffusion-weighted imaging (Charles-Edwards et al, 2008), as the latter provides a different contrast mechanism (Charles-Edwards and deSouza, 2006). However, at 3.0-T, the improvement in SNR over 1.5-T is offset by image artefacts on diffusion-weighted images due to magnetic field inhomogeneity (Merkle and Dale, 2006; Dietrich et al, 2008), which can be substantial when an endovaginal receiver coil is present. A ZOnal Oblique Multislice (ZOOM)-diffusion-weighted imaging (DWI) sequence addresses this by reducing the field of view and suppressing the signal from outside the volume of interest (Wilm et al, 2007; Wilm et al, 2009). ZOOM-DWI techniques have been used to image the spinal cord and the optic nerve (Wilm et al, 2007; Wilm et al, 2009) but have not been used to image the cervix. The aim of this study, therefore, was to determine the sensitivity and specificity of T2-W + ZOOM-DW endovaginal MRI (evMRI) at 3.0-T for detecting small volume cervical cancer in comparison with previously published values, measure the tumour-to-stromal contrast of T2-W and ZOOM-DWI to evaluate their contribution in tumour detection and to establish the effect of the endovaginal imaging information on surgical management of patients.

\section{MATERIALS AND METHODS}

Patients. The study was approved by the local institutional review board. All patients gave informed, written consent. Over a 14month period (March 2011-April 2012), 57 consecutive patients aged $25-66$ years (mean $=36.6 \pm 10.1$ years, 49 premenopausal) with suspected stage I cervical cancer who had presented following an abnormal smear test $(n=39)$ or with irregular bleeding/ abnormal vaginal discharge $(n=18)$ and with histologically documented invasive cancer (34 squamous cell carcinoma, 22 adenocarcinoma and 1 adenosquamous) were prospectively recruited for assessment with endovaginal T2-W MRI + ZOOMDWI at 3.0-T. At examination under anaesthesia (EUA) done after evMRI, 47 were staged as Ib1, 4 as Ib2 and 3 as IIb. Three proceeded to chemoradiotherapy without EUA on the basis of clinical examination and evMRI. Thirty-one patients were being considered for radical vaginal trachelectomy (RVT) based on the results of histology from cone biopsy/large loop excision of the transformation zone (LLETZ) and clinical examination; criteria for consideration included absolute desire for fertility-sparing surgery so as to retain their child-bearing potential and tumours $<2 \mathrm{cms}$ in diameter potentially with a clearance of at least $1 \mathrm{~cm}$ from the internal os. All patients had a biopsy at an average of 5 weeks (s.d. \pm 2.5 weeks, range $0.4-12.7$ weeks) before MRI in which some or the entire tumour may have been excised; however, all cone biopsies/LLETZ samples were positive to margins. Nineteen patients had undergone MRI at the time of referral done at other institutions using conventional external phased-array techniques. The presence or absence of tumour on these images was formally recorded by an independent specialist gynaecological radiologist at the tumour board before evMRI.

Imaging methods. Endovaginal images were obtained at 3.0-T (Philips Achieva, Best, The Netherlands) using a $37-\mathrm{mm}$ ringdesign solenoidal receiver coil similar to one previously described for use at 1.5-T (Charles-Edwards et al, 2008). Hyoscine butyl bromide (Buscopan, Boehringer Ingelheim $\mathrm{GmbH}$, Ingelheim, Germany) $20 \mathrm{mg}$ IM was administered to reduce artefact from bowel motion.

T2-weighted images and slice-matched ZOOM-DWI were acquired in three orthogonal planes to cover the cervix (Table 1). Isotropic apparent diffusion coefficient (ADC) maps based on monoexponential rate of signal decay with increasing diffusion weighting were generated with the system software (Philips Medical Systems, Best, The Netherlands) using all $b$-values. Following endovaginal imaging, the internal receiver coil was removed and large field of view images through the abdomen and pelvis were obtained for the assessment of lymph node status, but this did not form part of this study.

\section{Data analysis}

Sensitivity and specificity. T2-weighted and ZOOM-DWI images were assessed in combination for the presence of tumour by two separate observers (observer 1: 19 years experience of endovaginal MR imaging, observer 2: 2 years experience) without access to the details of previous histopathology. The presence of tumour was recorded if an intermediate signal-intensity focal mass on $\mathrm{T} 2-\mathrm{W}$ imaging corresponding to a focal area of diffusion restriction on

\begin{tabular}{|c|c|c|}
\hline Parameter & T2-weighted & Zoom-DWI \\
\hline Coil selection & $\begin{array}{l}\text { Dual: endovaginal + FLEX-L } 2 \\
\text { element phased-array body }\end{array}$ & Single: endovaginal \\
\hline Sequence & $\begin{array}{l}\text { Turbo spin echo-multi shot } \\
\text { (TSE factor 10) }\end{array}$ & $\begin{array}{l}\text { Spin-echo echo planar } \\
\text { imaging-single shot }\end{array}$ \\
\hline TR & $\begin{array}{l}2750 \mathrm{~ms} \text { (coronal) } \\
2500 \mathrm{~ms} \text { (sagittal, axial) }\end{array}$ & $\begin{array}{l}6500 \mathrm{~ms} \text { (coronal) } \\
5500 \mathrm{~ms} \text { (sagittal, axial) }\end{array}$ \\
\hline TE & $80 \mathrm{~ms}$ & $52 \mathrm{~ms}$ \\
\hline Flip angle & $90^{\circ}$ & $90^{\circ}$ \\
\hline FOV & $100 \mathrm{mn}$ & \\
\hline Bandwidth & 217.7 & 11.4 \\
\hline Matrix & $240 \times 288$ & $80 \times 224$ \\
\hline Pixel size & $0.42 \mathrm{~mm}$ & $1.25 \mathrm{~mm}$ \\
\hline Voxel size & $\begin{array}{l}0.36 \mathrm{~mm}^{3} \text { (acquisition) } \\
0.25 \mathrm{~mm}^{3} \text { (recon) }\end{array}$ & $\begin{array}{l}3.15 \mathrm{~mm}^{3} \text { (acquisition) } \\
0.41 \mathrm{~mm}^{3} \text { (recon) }\end{array}$ \\
\hline Slice thickness & $2 \mathrm{~mm}(0.1 \mathrm{~mm} \mathrm{~s}$ & paration) \\
\hline Number of slices & $\begin{array}{l}24 \text { (coronal) } \\
22 \text { (sagittal) } \\
20 \text { (axial) }\end{array}$ & \\
\hline $\begin{array}{l}\text { Number of signal } \\
\text { averages }\end{array}$ & 2 & 1 \\
\hline $\begin{array}{l}\text { b-values, } \mathrm{smm}^{-2} \\
\text { isotropic }\end{array}$ & NA & $\begin{array}{l}0,100,300,500,800 \\
\text { (coronal) } \\
0,500,800 \text { (sagittal, } \\
\text { axial) }\end{array}$ \\
\hline Acquisition time & $\begin{array}{l}4 \min 18 \mathrm{~s} \text { (coronal) } \\
4 \min 0 \mathrm{~s} \text { (sagittal) } \\
3 \min 55 \mathrm{~s} \text { (axial) }\end{array}$ & $\begin{array}{l}4 \min 33 \mathrm{~s} \text { (coronal) } \\
2 \min 21 \mathrm{~s} \text { (sagittal) } \\
2 \min 12 \mathrm{~s} \text { (axial) }\end{array}$ \\
\hline \multicolumn{3}{|c|}{$\begin{array}{l}\text { Abbreviations: } N A=\text { not applicable; } T E=\text { echo time; } T R=\text { repetition time; } Z O O M-D W I= \\
\text { ZOnal Oblique Multislice-diffusion-weighted imaging. }\end{array}$} \\
\hline
\end{tabular}


ADC maps derived from ZOOM-DWI was identified. Following identification of the tumour, its volume was recorded by observer 1 drawing a region-of-interest (ROI) around it on every slice on which it was identified on the T2-W images and multiplying the summed area of the ROIs by slice thickness. In patients with tumours $>1 \mathrm{~cm}^{3}$ in volume, the presence or absence of parametrial extension was also documented as we have previously observed parametrial extension on MRI or histology only on tumours of $>1 \mathrm{~cm}^{3}$ (Payne et al, 2010). To assess intraobserver agreement, observer 1's original reports recording the presence or absence of tumour and issued before surgical intervention were used for comparison. All findings were compared with the results at subsequent histopathology of the surgical specimen.

Tumour contrast. In patients with identifiable tumour, tumour contrast normalised to the cervix $\left(\left(S_{1}-S_{2}\right) / S_{2}\right)$ was calculated from ROIs within tumour $\left(\mathrm{S}_{1}\right)$ and adjacent normal cervical stroma $\left(\mathrm{S}_{2}\right)$ on a single slice of T2-W, corresponding ZOOM-DW image and ADC map. The images with the highest diffusion-weighting $\left(b=800 \mathrm{~s} \mathrm{~mm}^{-2}\right)$ were selected because retained signal at this $b$-value was probably to best reflect tumour-stromal contrast on ADC maps. Regions of interest encompassed the entire tumour and at least $10 \mathrm{~mm}^{2}$ of normal stroma; placement in relation to anatomy was performed by visual matching between T2-W and ZOOM-DWI. Regions of interest were copied from T2-W onto ZOOM-DWI images and adjusted to account for distortion. Care was taken to ensure that stromal ROIs were sited identically in relation to the endovaginal coil on both T2-W and ZOOM-DW images.

Decision for trachelectomy. Patients referred for RVT on the basis of histology of the cone biopsy/LLETZ specimen and clinical examination were reviewed at the multidisciplinary team meeting, and a decision for RVT $v s$ more or less radical treatment options was made on clinical (age $<45$ years, without evidence of impaired fertility and wishing to retain fertility) and imaging grounds (lesion $<4 \mathrm{~cm}$, no evidence of parametrial extension or metastasis and at least $8 \mathrm{~mm}$ from the superior aspect of the tumour to the internal os (Abu-Rustum et al, 2008)). The decision and reasoning was noted in the electronic patient record and transferred to the research file, recording whether or not the endovaginal imaging result was the primary reason for a change from the initial referral for RVT.

Histopathological analysis. Tissue was acquired at radical hysterectomy $(n=19)$, RVT $(n=15)$, cold knife cone $(n=12)$ or LLETZ procedure $(n=1)$ in 47 patients. The remaining 10 patients had no post-MRI histology for comparison because 9 of them required chemoradiotherapy and 1 had no further surgery as the tumour was subsequently found to be completely excised at the initial knife cone biopsy. All specimens were fixed in formalin. For hysterectomy and RVT specimens, a shave of the proximal endocervical resection margin (at or near the internal os) was sliced longitudinally (sagittal plane centrally, coronal plane laterally). The cervix together with the parametrium was sliced into transverse sections up to $\sim 1 \mathrm{~cm}$ from the ectocervix. The remaining $1 \mathrm{~cm}$ of distal cervix together with the vaginal cuff was sectioned longitudinally (sagittal plane centrally, coronal plane laterally). All slices (each 3-4 mm thick) were processed and embedded in paraffin; $2-3 \mu \mathrm{m}$ sections were deparaffinised and stained with haematoxylin and eosin. According to the thickness of the slice obtained at macroscopy, each histological slide was separated from the next by $3-4 \mathrm{~mm}$.

Statistical analysis. Sensitivity, specificity, positive and negative predictive values of $\mathrm{T} 2-\mathrm{W}$ combined with ZOOM-DWI in distinguishing the presence of tumour was established for two independent observers and the level of agreement between them was calculated using kappa statistics. This was compared with that of standard imaging done at the referring centres. In patients with definite radiological and histological evidence of tumour (true positives), contrast between tumour and adjacent stromal regions normalised to stromal values were compared between T2-W and ZOOM-DWI and between tumour and stromal ADC values using a paired $t$-test. A $P$-value of $<0.05$ was used to indicate significance.

\section{RESULTS}

Detection and local staging of small volume disease. Forty-seven of 57 patients underwent a surgical procedure a median of 5 weeks of MRI (interquartile range 3-8 weeks) and therefore had histology for comparison; of these, 24 had residual tumour and 23 had no residual tumour on histology. Sensitivity and specificity for detecting tumour on endovaginal T2-W + ZOOM DWI in 47 cases are given in Table 2 and a true-positive lesion is illustrated in Figure 1. There were four false positives (median volume $0.04 \mathrm{~cm}^{3}$, interquartile range $0.03-0.05 \mathrm{~cm}^{3}$, (Figure 2)) and two false negatives (Figure 3 ) reported by observer 1 , and six false positives (median volume $0.20 \mathrm{~cm}^{3}$, interquartile range $0.05-0.32 \mathrm{~cm}^{3}$ ) and four false negatives reported by observer 2 . In comparison, truepositive lesions had median volumes of $1.70 \mathrm{~cm}^{3}$ for both observers (interquartile range $0.3-2.9 \mathrm{~cm}^{3}$ for observer 1 and $0.5-5.4 \mathrm{~cm}^{3}$ for observer 2). The interobserver agreement was good (kappa $=0.65)$ and intraobserver agreement was excellent (kappa $=0.89)$. When tumours $<0.1 \mathrm{~cm}^{3}$ on histology were omitted from the analysis (which equates to a 5-mm histological dimension on a 4-mm-thick section (Charles-Edwards et al, 2011), the specificity improved to $100 \%$ (observer 1) and 80\% (observer 2). Of 19 patients with previous external imaging, 5 were positive for tumour (also noted on evMRI and confirmed on histology) and 14 were negative (sensitivity $50 \%$, specificity $100 \%$ ). In these patients, evMRI had a sensitivity of $100 \%$ and specificity of $88.8 \%$.

The presence or absence of parametrial extension was documented in tumours over $1 \mathrm{~cm}^{3}(n=14$ patients with 28 regions assuming right and left parametrial extension are effectively independent). Parametrial extension was detected with a sensitivity of $50 \%$ but a specificity of $100 \%$ for both observers (positive predictive value $100 \%$, negative predictive value $88 \%$ ). There was complete agreement between observers (kappa $=1)$. In the three false-negative regions $(n=2$ patients), the parametrial extension was $1 \mathrm{~mm}$ (tumour volume on MRI, $5.6 \mathrm{~cm}^{3}$ ), and 2 and $3 \mathrm{~mm}$ (tumour volume on MRI $2.0 \mathrm{~cm}^{3}$ ), and the time interval between MRI and surgery (radical hysterectomy in both cases) was 22 days and 45 days, respectively; tumour growth during this period was not accounted for.

Tumour-to-stromal contrast. Tumour was noted in 28 of 57 patients; 20 had subsequent histological confirmation of tumour, whereas 8 had pre-procedural histological evidence of tumour but because of imaging and EUA evidence of parametrial extension $(n=7)$ or lymph node metastases $(n=1)$ they were treated with

\begin{tabular}{|c|c|c|c|c|}
\hline & $\begin{array}{c}\text { Sensitivity } \\
(\%)\end{array}$ & $\begin{array}{c}\text { Specificity } \\
(\%)\end{array}$ & $\begin{array}{l}\text { PPV } \\
(\%)\end{array}$ & $\begin{array}{l}\text { NPV } \\
(\%)\end{array}$ \\
\hline Observer 1 reporting read & 88.0 & 81.8 & 84.6 & 85.7 \\
\hline Observer 1 anonymised read & 92.0 & 81.8 & 85.2 & 90.0 \\
\hline Observer 2 anonymised read & 84.0 & 72.7 & 77.8 & 80.0 \\
\hline
\end{tabular}



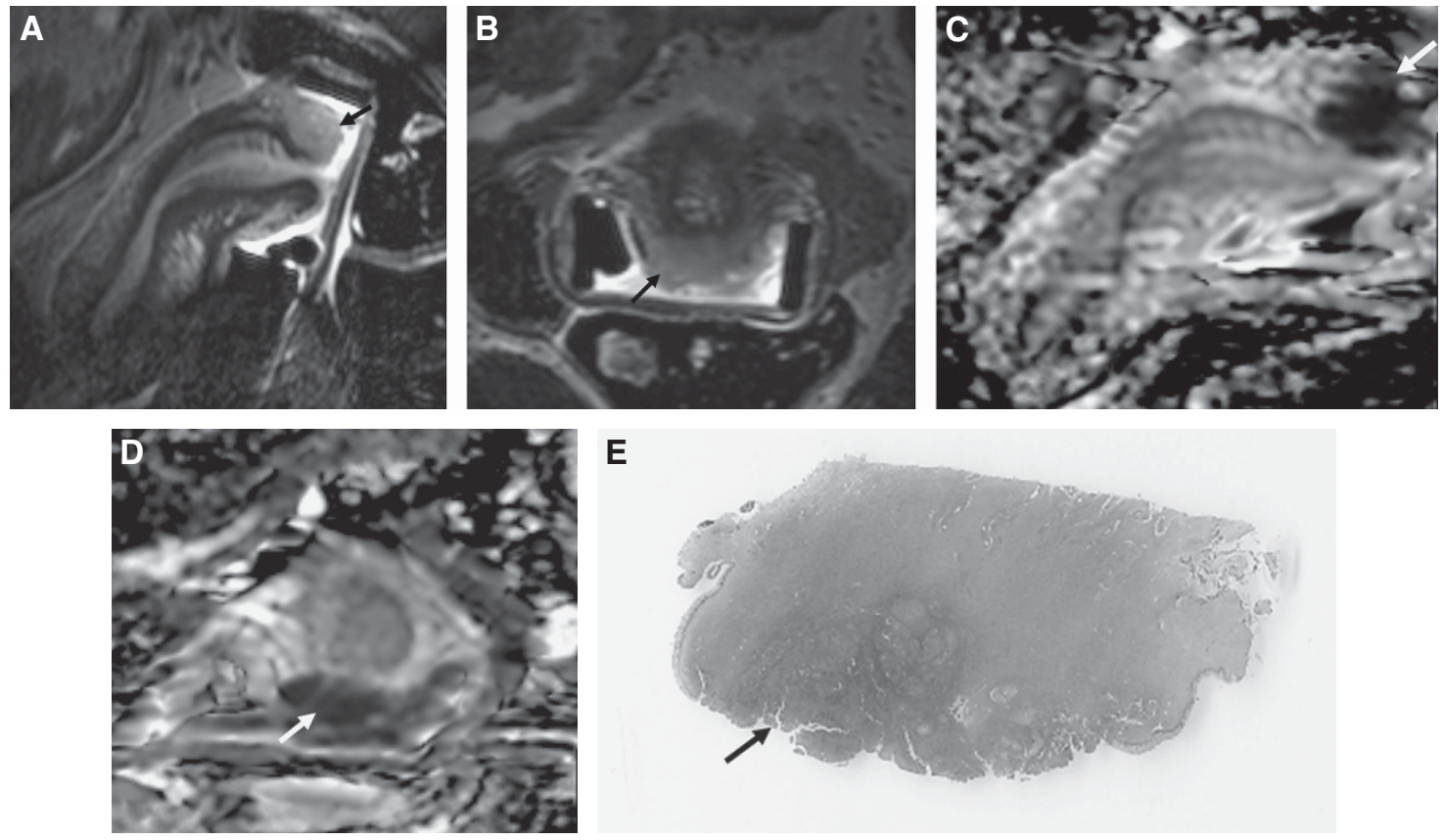

E

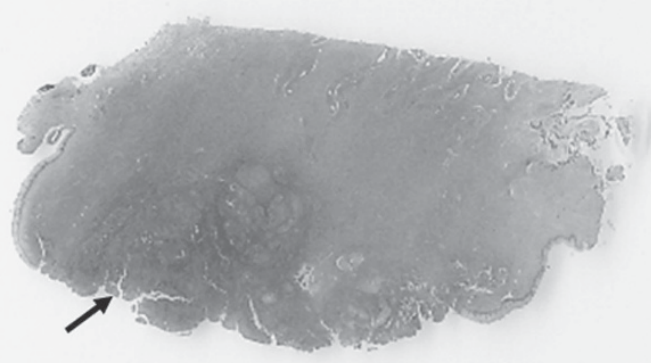

Figure 1. True positive in a 25 -year-old female diagnosed with stage I carcinoma of the cervix following intermittent postcoital bleeding. T2-W images in the sagittal (A) and coronal (B) planes orthogonal to the cervix show a small cervical tumour (arrows). The ADC maps generated from ZOOM-DWI in corresponding orientations ( $\mathbf{C}$, sagittal and $\mathbf{D}$, coronal) show that the tumour (arrows) is more easily appreciated in $\mathbf{C}$ and $\mathbf{D}$, although considerable image distortion is present. This mandates the use of the T2-W images for anatomical delineation. Good correspondence is seen with the histological definition of the tumour (dark blue) on the haematoxylin and eosin-stained corresponding trachelectomy specimen (E, arrow).
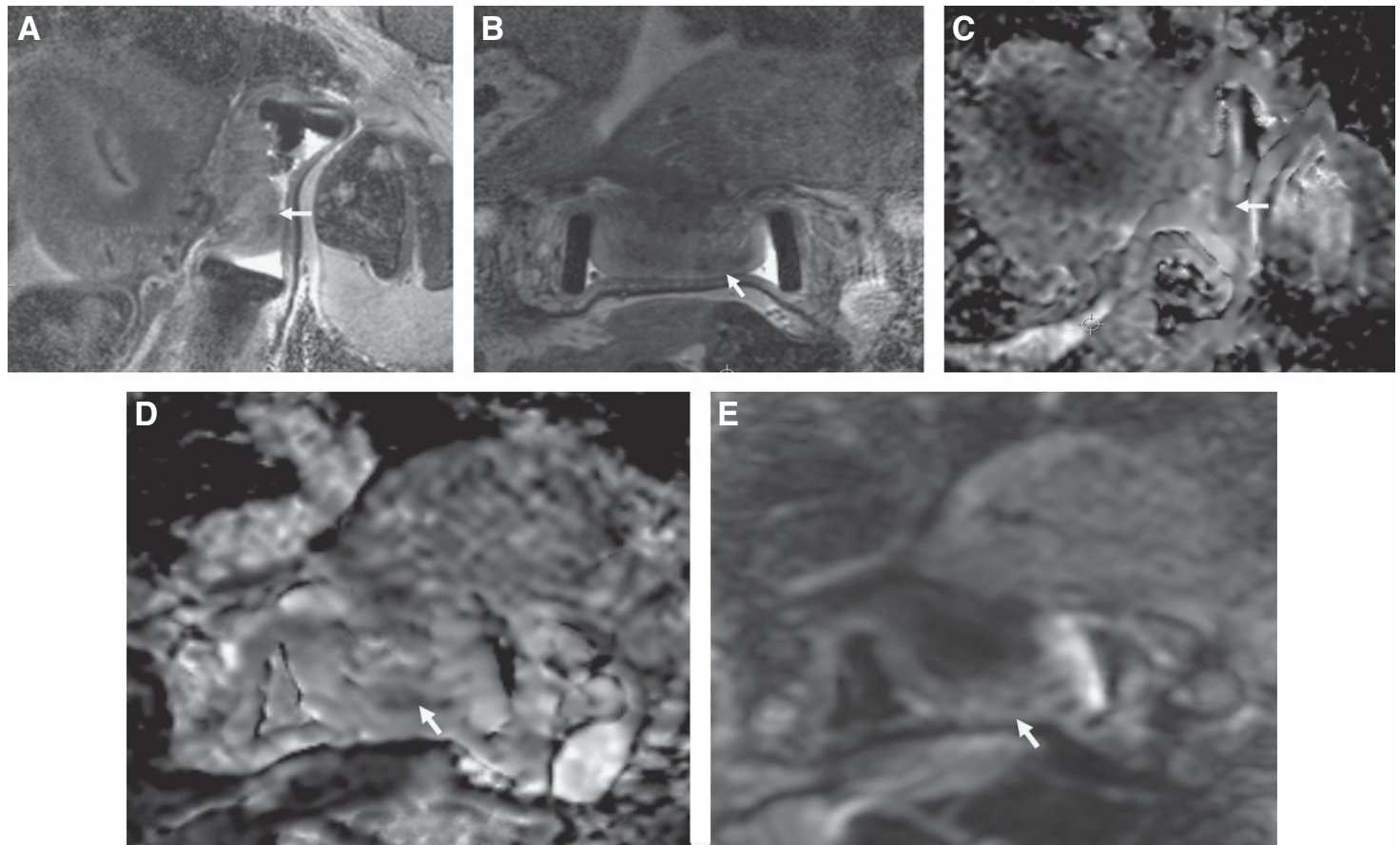

Figure 2. False positive in a 33-year-old female diagnosed with stage I carcinoma of the cervix following positive smear test and cone biopsy done 2 weeks before MRI. T2-W images in the sagittal (A) and coronal (B) planes orthogonal to the cervix show an ill-defined area of intermediate signal intensity (arrows). This lesion demonstrates diffusion restriction (arrows) on the corresponding ADC maps in corresponding orientations (C, sagittal and $\mathbf{D}$, coronal). The coronal $b=800 \mathrm{~s} \mathrm{~mm}^{-2}(\mathbf{E})$ is also illustrated for comparison. There was no residual tumour on the trachelectomy specimen.

subsequent chemoradiotherapy. Contrast between tumour and adjacent normal cervical stroma on T2-W imaging in these 28 cases was $1.39 \pm 0.95$ compared with $3.30 \pm 2.83$ on ZOOM-DWI, being significantly greater in the ZOOM-DWI $(P<0.00004)$ compared with T2-W images (Figure 4). The ADC values for these lesions ranged from 657 to $1430 \times 10^{-6} \mathrm{~mm}^{2} \mathrm{~s}^{-1}$ 

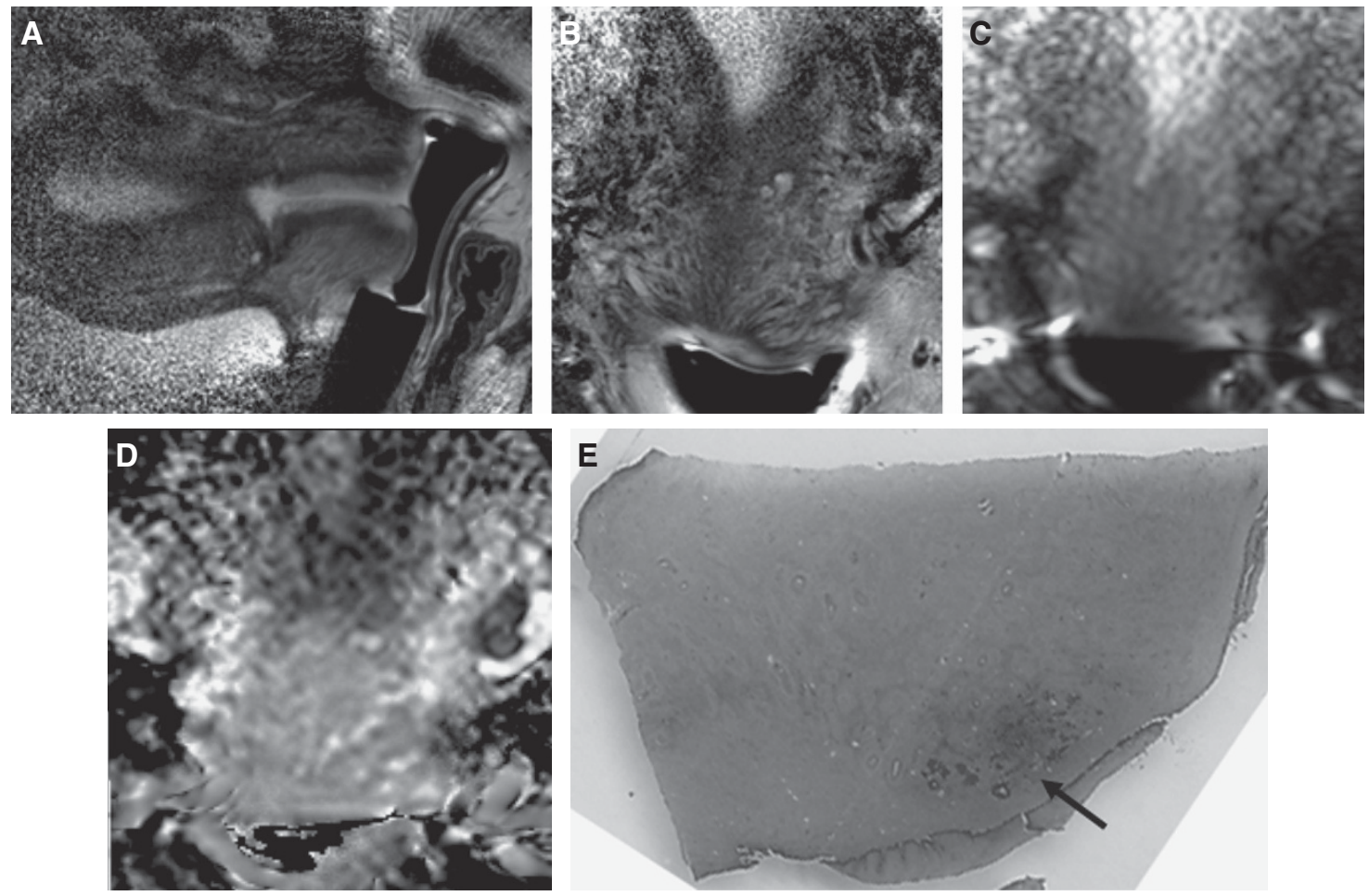

Figure 3. False negative in a 35-year-old female diagnosed with stage I carcinoma of the cervix following an abnormal smear test. T2-W images in the sagittal (A) and coronal (B) planes orthogonal to the cervix with corresponding coronal ZOOM-DWI (C) and coronal ADC map (D). There was no evidence of tumour on the assessment of the T2-W + ZOOM-DWI in combination. At trachelectomy (haematoxylin and eosin-stained section, E), a 3.9-mm tumour focus was demonstrated (arrow).

(mean \pm s.d., $906 \pm 182 \times 10^{-6} \mathrm{~mm}^{2} \mathrm{~s}^{-1}$ ) and were significantly different from stromal values of 1276 to $1937 \times 10^{-6} \mathrm{~mm}^{2} \mathrm{~s}^{-1}$ (mean \pm s.d., $\quad 1551 \pm 209 \times 10^{-6} \mathrm{~mm}^{2} \mathrm{~s}^{-1}, \quad P<0.000001$ ). Phantom quality assurance data done before each patient study showed that signal-to noise ratio within the coil ring from where measurements of contrast were derived was $501 \pm 97$, indicating that signal in this region was well above the noise floor, confirming the validity of the contrast measurements.

Effect of evMRI on the decision for trachelectomy. In the subgroup of 31 patients referred for RVT, 17 patients had no visible tumour on the endovaginal imaging (observer 1). Nine were therefore treated with less radical surgery (extended cone biopsy) and 1 patient had no treatment because of complete excision of early microinvasive disease $(0.2 \mathrm{~mm}$ wide, $0.2 \mathrm{~mm}$ in depth with no lymphovascular space invasion (LVSI)) at LLETZ before MRI. Cone biopsy was performed where the tumour was $<1 \mathrm{~cm}$ and upto $5 \mathrm{~mm}$ depth as the aim is conservation of cervical stroma where possible. Six proceeded to RVT (following negative lymph nodes at dissection) based on histology that showed a combination of stage $1 \mathrm{~b}$ disease, LVSI positivity, additional small focus of synchronous SCC on the upper vagina and narrow excision margins at previous diagnostic cone biopsy. The final patient was treated with chemoradiotherapy because of a lymph node metastasis on the pelvic node dissection that was not detected on imaging. Histology in these cases $(n=16$ because of the lack of histology from the primary site in the patient treated with chemoradiotherapy) confirmed the absence of tumour in 15 cases (14 post-MRI and 1 on review of the pre-MRI cone biopsy), whereas 1 patient was falsely negative (maximum tumour dimensions on subsequent histology was $3.9 \mathrm{~mm}$; Figure 3).

Fourteen of 31 patients had visible tumour on MRI (observer 1); 9 cases were deemed suitable for RVT and, based on MRI measurements of length of normally appearing endocervical canal above tumour (Table 3), proceeded to this surgical option (2 were falsely positive). One patient was considered suitable for extended cone biopsy and one had a radical hysterectomy (because of the presence of extensive pelvic endometriosis and multiple fibroids rendering the patient unsuitable for RVT). Three patients had chemoradiotherapy because parametrial extension was demonstrated on evMRI in two patients and a lymph node at pelvic dissection was positive in the other. Thus, overall the evMRI itself dictated the use of less radical surgery than RVT (extended cone biopsy) in 10 out of $31(32.3 \%)$ cases and more radical treatment than RVT (chemoradiotherapy) in 2 out of $31(6.5 \%)$ cases.

A flow chart of patient referral and subsequent management is detailed in Figure 5.

\section{DISCUSSION}

These results indicate that $\mathrm{T} 2-\mathrm{W}+\mathrm{ZOOM}-\mathrm{DWI}$ has a high sensitivity and specificity for detecting tumours clinically staged as $1 \mathrm{a} / 1 \mathrm{~b} 1$ disease primarily because of high tumour-to-stromal contrast on ZOOM-DWI compared with T2-W MRI, and that its use in patients referred for RVT alters the surgical management in up to $39 \%$ of cases. Decision for extended cone biopsy or RVT (excision of the cervix taking a parametrial and a proximal vaginal cuff with a vagino-isthmic anastomosis (Dargent et al, 1994; Covens et al, 1999)) requires an accurate preoperative delineation of the size, extent and location of tumour within the cervix. Previously, MRI has been used for evaluating endocervical extension and patient suitability for RVT where extension to the internal os was seen in a quarter of cases in a single small series (Peppercorn et al, 1999). Although the decision for extended cone biopsy or RVT is multifactorial and includes histological and clinical factors, we recorded the cases in which imaging primarily 

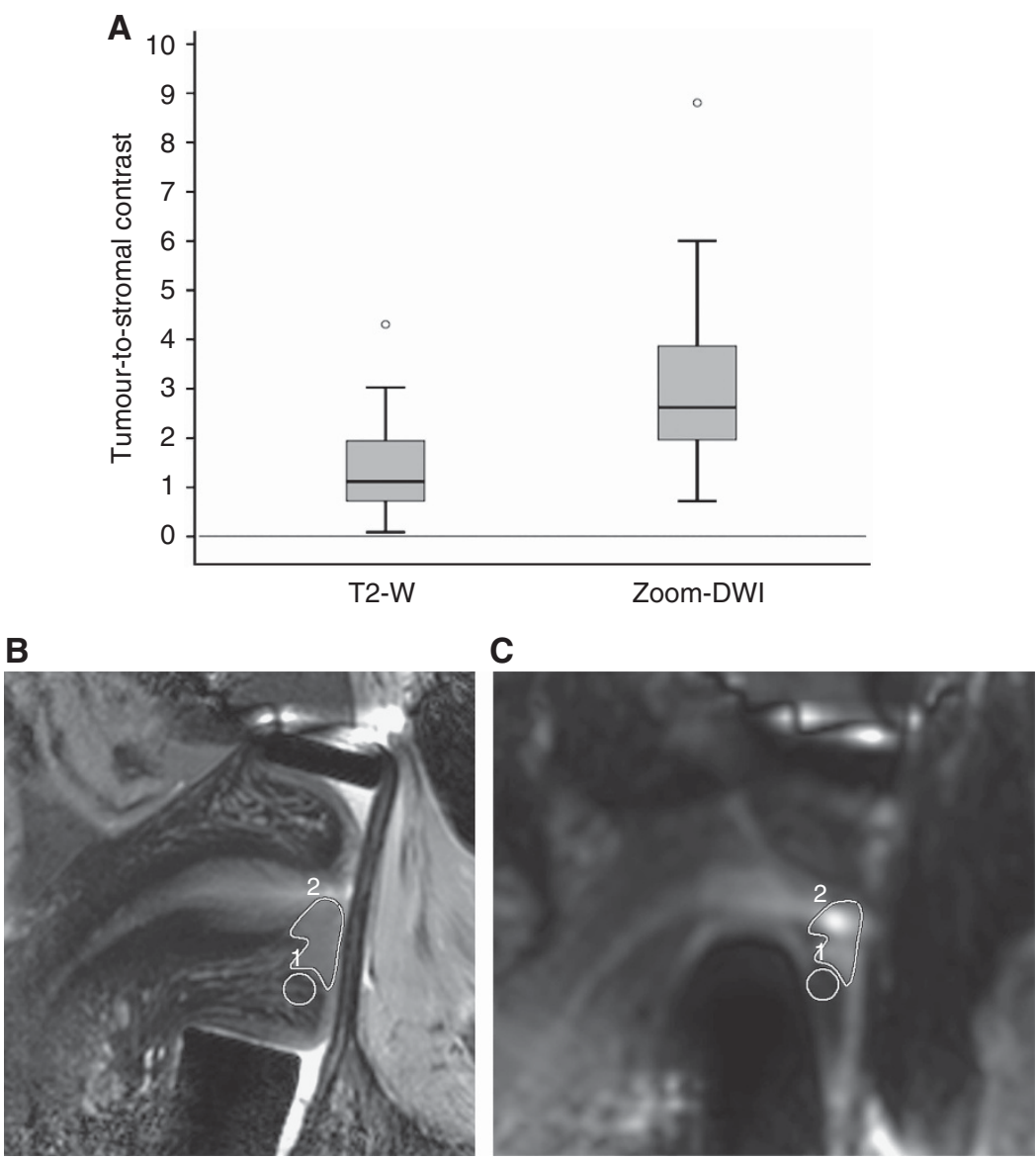

Figure 4. Tumour-to-stromal contrast of cervical cancer on T2-W and ZOOM-DWI. Boxplot of tumour-to-stromal contrast in T2-W compared with ZOOM-DWI images (A). The line at a normalised tumour-to-stromal signal-intensity of 0 indicates no contrast. Example ROls delineated around tumour (1) and adjacent stroma (2) on sagittal T2-W (B), and corresponding ZOOM-DWI b=800 s mm ${ }^{-2}$ (C) images from which these data are derived.

Table 3. Imaging measurements in patients with endovaginal images positive for tumour that proceeded to radical vaginal trachelectomy

\begin{tabular}{|c|c|c|c|c|}
\hline $\begin{array}{l}\text { Patient } \\
\text { number }\end{array}$ & $\begin{array}{c}\text { Tumour volume }\left(\mathbf{c m}^{\mathbf{3}}\right)(\mathbf{m a x i m u m} \\
\text { dimension }(\mathbf{m m}))\end{array}$ & $\begin{array}{c}\text { Depth of stromal } \\
\text { invasion }(\mathbf{m m})\end{array}$ & $\begin{array}{c}\text { Distance from superior tumour margin } \\
\text { to internal os (mm) }\end{array}$ & $\begin{array}{c}\text { Histology for } \\
\text { presence of tumour }\end{array}$ \\
\hline 1 & $2.1(16)$ & 11 & 20 & Positive \\
\hline 16 & $0.5(11)$ & 12 & 16 & Positive \\
\hline 23 & $2.0(22)$ & 13 & 20 & Positive \\
\hline 38 & $0.3(15)$ & 11 & 21 & Positive \\
\hline 39 & $0.03(8)$ & 2 & 19 & Negative \\
\hline 50 & $0.04(7)$ & 2 & 9 & Negative \\
\hline 52 & $0.4(15)$ & 15 & 17 & Positive \\
\hline 55 & $0.3(15)$ & 7 & 10 & Positive \\
\hline 56 & $1.5(18)$ & 12 & & Positive \\
\hline
\end{tabular}

influenced the decision. Review of the final histology, which was negative in all 10 patients in whom a decision for extended cone was based on evMRI findings, indicates that our imaging assessment was correct.

Endovaginal MRI at 3.0-T correctly identified tumour in more than a third of cases with negative external-array imaging. Our results also show that the sensitivity and specificity for detection of tumour when using T2-W in conjunction with ZOOM-DWI is better than previous data collected at 1.5-T (Charles-Edwards et al, 2008 ) both for an experienced $(92.0 \%$ and $81.8 \%$, respectively compared with $88.9 \%$ and $66.7 \%$, respectively) as well as a less experienced $(84.0 \%$ and $72.7 \%$ compared with $83.3 \%$ and $69.6 \%$, respectively) observer. The difference in sensitivity and specificity between observers was related to the subcentimeter tumour voume in an $\sim 60 \%$ of positive cases. The lower sensitivity for observer 1 at the time of reporting is attributed to one case with an $18-\mathrm{mm}^{3}$ focus of disease on the histology initially scored negative. Falsepositive results had volumes $<0.2 \mathrm{~cm}^{3}$ and were likely to represent areas of granulation tissue after biopsy that are indistinguishable from invasive cancer on T2-W imaging. False-negative tumours 


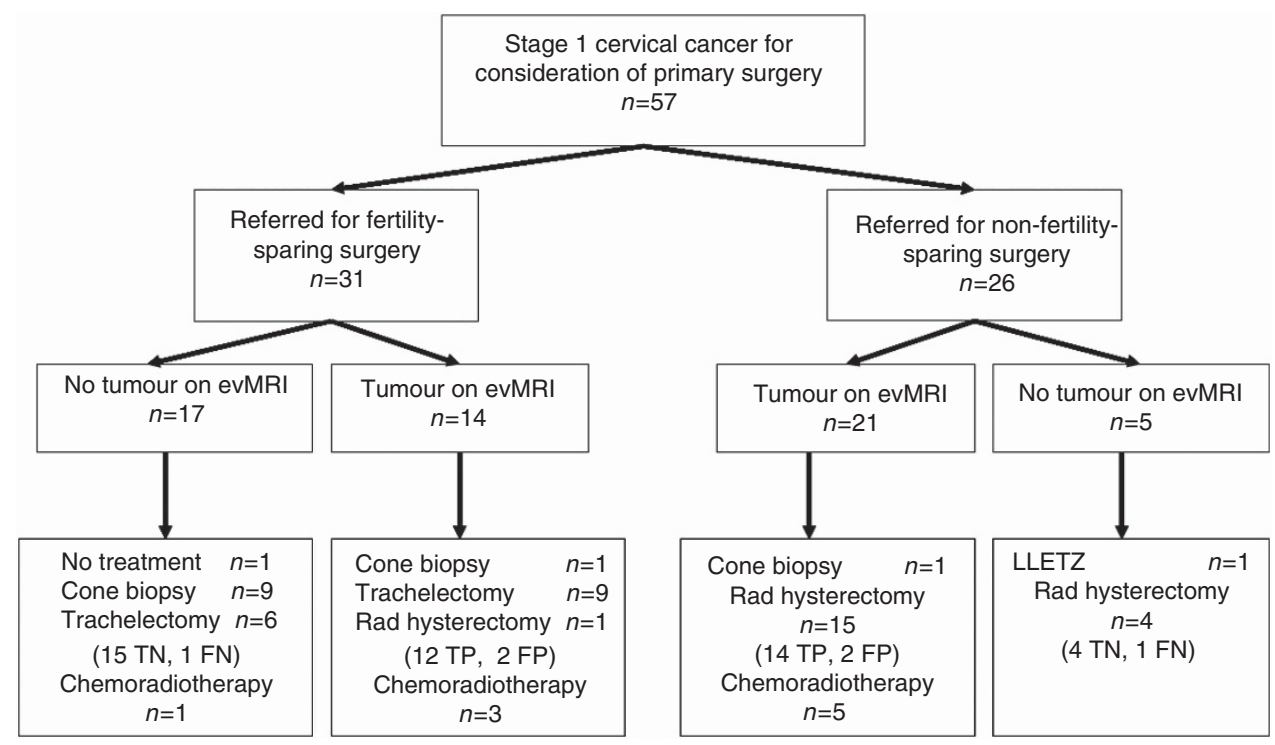

Figure 5. Flow chart of patients referred for assessment, evMRI findings and subsequent management.

were also small in size; the largest tumour length dimension on histology missed by both observers 1 and 2 was $6.9 \mathrm{~mm}$ and may have been obscured by artefact or simply beyond the resolution of the technique, particularly as it was a thin plaque-like lesion.

Sensitivity for parametrial extension was low because of the very early invasion in a relatively small patient cohort. In all three false-negative parametrial regions, the parametrial extension was documented as $<3 \mathrm{~mm}$, which was the dimension of two ZOOM-DWI pixels. In patients with obvious parametrial extension detected on MRI, treatment was with chemoradiation rather than surgery $(n=7)$, thus excluding them from the histological comparison; unfortunately, ethical considerations would prevent these patients from being treated with primary surgery. Sensitivity for parametrial extension therefore was lower than expected in comparison with previous early studies, where primary surgery was utilised more commonly in bulky stage Ib2 disease (Subak et al, 1995; Sheu et al, 2001; deSouza et al, 2006). Further improvements in spatial resolution and image distortion are needed to improve the sensitivity of detection of parametrial extension of $<3 \mathrm{~mm}$.

A comparison was not made in this study of the sensitivity to detect tumour with the endovaginal coil compared with external array imaging only. Although repeating the scans without an endovaginal coil for comparison would have been ideal, performing an optimal external phased-array imaging after the endovaginal imaging (or vice versa) was not achievable because of increased peristalsis from after-effects of antiperistaltic agents, bladder filling and increased patient motion with a lengthy protocol. Repeating the examination on a separate occasion would have met with poor compliance. In 19 patients where external imaging was available, $\sim 36 \%$ of lesions not visible on referral scans were correctly identified on the endovaginal imaging.

The improvement in sensitivity of cervical tumour detection by adding conventional DWI to T2-W sequences has previously been documented at 1.5-T (Charles-Edwards et al, 2008). The use of $b$-values of $b-800 \mathrm{~s} \mathrm{~mm}^{-2}$ and $b-1000 \mathrm{~s} \mathrm{~mm}^{-2}$ have been recommended for DWI of the female pelvis (Koyama and Togashi, 2007) because they allow optimum visualisation of tumour with suppression of normal background structures. Selection of $b=800 \mathrm{~s} \mathrm{~mm}^{-2}$ was based on published values of ADC for cervical tumour between 0.9 and $1.2 \times 10^{-3} \mathrm{~mm}^{2} \mathrm{~s}^{-1}$ (Hoogendam et al, 2010; Fu et al, 2012) and because $b>1000 \mathrm{~s} \mathrm{~mm}^{-2}$ suffer from poor SNR (Issa, 2002; Naganawa et al, 2005).
Although we viewed the ADC maps rather than the high $b$-value images, the information they provided was equivalent; our use of the ADC maps reflected local practice. However, the utility of the T2-W images themselves for depicting morphology, differentiating zonal anatomy and the boundaries between the cervix and the parametrium should not be underestimated. We have previously shown that the improvement in contrast with DWI needs to be viewed in context of the anatomical detail (Charles-Edwards et al, 2008).

The use of the endovaginal coil did not incur additional specific absorbtion rate and was equivalent to values for endorectal coil imaging of the prostate. However, in the presence of an endovaginal coil at 3.0-T, the use of ZOOM-DWI was essential to achieve usable DW contrast, as standard diffusion-weighted sequences resulted in such severe image distortion so as to render the images unusable clinically; their implementation for comparison with ZOOM-DWI was therefore ethically not justifiable. However, despite the use of ZOOM-DWI, distortion remains an issue, although suppression of signal outside the volume of interest minimised the image-wrapping artefacts prevalent in all echoplanar sequences and inclusion of a reverse gradient technique partly corrected the inherent field inhomogeneity (Volk et al, 1987). Further work will explore software (multiecho TSE) solutions to reduce image distortion. Other factors reducing image quality are motion of the cervix during the examination. False-positive/-negative results from movement of the cervix due to bladder filling during the examination were a problem in some cases; an effective fluid restriction policy before imaging should help reduce this in future. In addition, air in the vagina and coil components have the potential to contribute significantly to image distortion because of $\mathrm{B} 1$ field inhomogeneity. We limited the former by suction extraction before imaging, but in many instances this was imperfect. To achieve more effective suction would require the incorporation of a suction mechanism into the coil itself. To address the latter, capacitors were sited in the handle away from the coil ring, but availability of non-ferromagnetic components in future should improve susceptibility artefact from coil electronics. Alternative hardware designs to a solenoidal ring would be needed for post-trachelectomy follow-up.

In summary, T2-W + ZOOM-DWI of the cervix using an endovaginal receiver coil at $3.0-\mathrm{T}$ had a high sensitivity and specificity for detecting cervical tumours clinically staged as Ia/Ib1 
primarily because of higher tumour-to-stromal contrast on ZOOM-DWI compared with T2-W MRI. In patients referred for RVT, the use of T2-W + ZOOM-DWI using an endovaginal coil is useful in preoperative assessment and potentially alters surgical management to a less or more radical approach in more than a third of cases.

\section{ACKNOWLEDGEMENTS}

We thank Dr DJ Gilderdale and Mr C Cummings for design and manufacture of the endovaginal coil. This work was supported by Medical Research Council Grant Number G0802470. The Imaging Centre is supported by CRUK \& EPSRC in association with MRC and Dept of Health C1060/A10334 and NHS funding to the NIHR Clinical Research Facility in Imaging and the Biomedical Research Centre.

\section{CONFLICT OF INTEREST}

The authors declare no conflict of interest.

\section{REFERENCES}

Abu-Rustum NR, Neubauer N, Sonoda Y, Park KJ, Gemignani M, Alektiar KM, Tew W, Leitao MM, Chi DS, Barakat RR (2008) Surgical and pathologic outcomes of fertility-sparing radical abdominal trachelectomy for FIGO stage IB1 cervical cancer. Gynecol Oncol 111: 261-264.

Bipat S, Van Den Berg RA, Van Der Velden J, Stoker J, Spijkerboer AM (2011) The role of magnetic resonance imaging in determining the proximal extension of early stage cervical cancer to the internal os. Eur J Radiol 78: 60-64.

Charles-Edwards E, Morgan V, Attygalle AD, Giles SL, Ind TE, Davis M, Shepherd J, Mcwhinney N, deSouza NM (2011) Endovaginal magnetic resonance imaging of stage $1 \mathrm{~A} / 1 \mathrm{~B}$ cervical cancer with $\mathrm{A} \mathrm{T} 2$ - and diffusion-weighted magnetic resonance technique: effect of lesion size and previous cone biopsy on tumor detectability. Gynecol Oncol 120: 368-373.

Charles-Edwards EM, deSouza NM (2006) Diffusion-weighted magnetic resonance imaging and its application to cancer. Cancer Imaging 6: 135-143.

Charles-Edwards EM, Messiou C, Morgan VA, De Silva SS, Mcwhinney NA, Katesmark M, Attygalle AD, deSouza NM (2008) Diffusion-weighted imaging in cervical cancer with an endovaginal technique: potential value for improving tumor detection in stage Ia and Ib1 disease. Radiology 249: 541-550.

Covens A, Shaw P, Murphy J, Depetrillo D, Lickrish G, Laframboise S, Rosen B (1999) Is radical trachelectomy a safe alternative to radical hysterectomy for patients with stage IA-B carcinoma of the cervix? Cancer 86: 2273-2279.

Dargent D, Brun J, Roy M, Remy I (1994) Pregnancies following radical trachelectomy for invasive cervical cancer. Gynecol Oncol 54: 105.

deSouza NM, Dina R, Mcindoe GA, Soutter WP (2006) Cervical cancer: value of an endovaginal coil magnetic resonance imaging technique in detecting small volume disease and assessing parametrial extension. Gynecol Oncol 102: $80-85$.

deSouza NM, Scoones D, Krausz T, Gilderdale DJ, Soutter WP (1996) High-resolution MR imaging of stage I cervical neoplasia with a dedicated transvaginal coil: MR features and correlation of imaging and pathologic findings. AJR Am J Roentgenol 166: 553-559.

deSouza NM, Whittle M, Williams AD, Sohail M, Krausz T, Gilderdale DJ, Mcindoe GA, Soutter WP (2000) Magnetic resonance imaging of the primary site in stage I cervical carcinoma: A comparison of endovaginal coil with external phased array coil techniques at 0.5T. J Magn Reson Imaging 12: 1020-1026.

Dietrich O, Reiser MF, Schoenberg SO (2008) Artifacts in 3-T MRI: physical background and reduction strategies. Eur J Radiol 65: 29-35.
Fu C, Bian D, Liu F, Feng X, Du W, Wang X (2012) The value of diffusionweighted magnetic resonance imaging in assessing the response of locally advanced cervical cancer to neoadjuvant chemotherapy. Int J Gynecol Cancer 22: 1037-1043.

Gien LT, Covens A (2010) Fertility-sparing options for early stage cervical cancer. Gynecol Oncol 117: 350-357.

Gilderdale DJ, deSouza NM, Coutts GA, Chui MK, Larkman DJ, Williams AD, Young IR (1999) Design and use of internal receiver coils for magnetic resonance imaging. Br J Radiol 72: 1141-1151.

Hoogendam JP, Klerkx WM, De Kort GA, Bipat S, Zweemer RP, Sie-Go DM, Verheijen RH, Mali WP, Veldhuis WB (2010) The influence of the b-value combination on apparent diffusion coefficient based differentiation between malignant and benign tissue in cervical cancer. J Magn Reson Imaging 32: 376-382.

Hricak H, Gatsonis C, Coakley FV, Snyder B, Reinhold C, Schwartz LH, Woodward PJ, Pannu HK, Amendola M, Mitchell DG (2007) Early invasive cervical cancer: CT and MR imaging in preoperative evaluation ACRIN/GOG comparative study of diagnostic performance and interobserver variability. Radiology 245: 491-498.

Issa B (2002) In vivo measurement of the apparent diffusion coefficient in normal and malignant prostatic tissues using echo-planar imaging. J Magn Reson Imaging 16: 196-200.

Koyama T, Togashi K (2007) Functional MR imaging of the female pelvis. J Magn Reson Imaging 25: 1101-1112.

Merkle EM, Dale BM (2006) Abdominal MRI at 3.0T: the basics revisited. AJR Am J Roentgenol 186: 1524-1532.

Naganawa S, Sato C, Kumada H, Ishigaki T, Miura S, Takizawa O (2005) Apparent diffusion coefficient in cervical cancer of the uterus: comparison with the normal uterine cervix. Eur Radiol 15: 71-78.

Payne GS, Schmidt M, Morgan VA, Giles S, Bridges J, Ind T, deSouza NM (2010) Evaluation of magnetic resonance diffusion and spectroscopy measurements as predictive biomarkers in stage 1 cervical cancer. Gynecol Oncol 116: 246-252.

Peppercorn PD, Jeyarajah AR, Woolas R, Shepherd JH, Oram DH, Jacobs IJ, Armstrong P, Lowe D, Reznek RH (1999) Role of MR imaging in the selection of patients with early cervical carcinoma for fertility-preserving surgery: initial experience. Radiology 212: 395-399.

Sahdev A, Sohaib SA, Wenaden AE, Shepherd JH, Reznek RH (2007) The performance of magnetic resonance imaging in early cervical carcinoma: a long-term experience. Int J Gynecol Cancer 17: 629-636.

Shepherd JH, Spencer C, Herod J, Ind TE (2006) Radical vaginal trachelectomy as a fertility-sparing procedure in women with early-stage cervical cancer-cumulative pregnancy rate in a series of 123 women. BJOG 113: 719-724.

Sheu MH, Chang CY, Wang JH, Yen MS (2001) Preoperative staging of cervical carcinoma with MR imaging: a reappraisal of diagnostic accuracy and pitfalls. Eur Radiol 11: 1828-1833.

Soutter WP, Hanoch J, D'arcy T, Dina R, Mcindoe GA, deSouza NM (2004) Pretreatment tumour volume measurement on high-resolution magnetic resonance imaging as a predictor of survival in cervical cancer. BJOG 111: $741-747$.

Subak LL, Hricak H, Powell CB, Azizi L, Stern JL (1995) Cervical carcinoma: computed tomography and magnetic resonance imaging for preoperative staging. Obstet Gynecol 86: 43-50.

Volk A, Tiffon B, Mispelter J, Jm L (1987) Chemical shift-specific slice selection. A new method for chemical shift imaging at high magnetic field. J Magn Reson 71: 168-174.

Wilm BJ, Gamper U, Henning A, Pruessmann KP, Kollias SS, Boesiger P (2009) Diffusion-weighted imaging of the entire spinal cord. NMR Biomed 22: 174-181.

Wilm BJ, Svensson J, Henning A, Pruessmann KP, Boesiger P, Kollias SS (2007) Reduced field-of-view MRI using outer volume suppression for spinal cord diffusion imaging. Magn Reson Med 57: 625-630.

This work is published under the standard license to publish agreement. After 12 months the work will become freely available and the license terms will switch to a Creative Commons AttributionNonCommercial-Share Alike 3.0 Unported License. 